\title{
The Composition of the Bulge Globular Cluster NGC 6273
}

\author{
C. A. Pilachowski ${ }^{1}$, C. I. Johnson ${ }^{2}$, R. M. Rich ${ }^{3}$, N. Caldwell ${ }^{2}$, \\ M. Mateo ${ }^{4}$, J. I. Bailey, III ${ }^{4}$ and J. D. Crane ${ }^{5}$ \\ ${ }^{1}$ Indiana University Bloomington \\ Department of Astronomy SW319 \\ 727 E 3rd St. \\ Bloomington, IN 47405 USA \\ email: cpilacho@indiana.edu \\ ${ }^{2}$ Harvard-Smithsonian Center for Astrophysics \\ 60 Garden St. MS-15 \\ Cambridge, MA 02138 USA \\ email: cjohnson@cfa.harvard.edu \\ ${ }^{3}$ University of California at Los Angeles \\ ${ }^{4}$ University of Michigan at Ann Arbor \\ ${ }^{5}$ The Observatories of the Carnegie Institution of Washington
}

\begin{abstract}
Observations of red giants in the Bulge globular cluster NGC 6273 with the Michigan/Magellan Fiber System (M2FS) mounted on the Nasmuth-East port of the Magellan-Clay 6.5-m telescope at the Las Campanas Observatory reveal a spread in metallicity. Members have been confirmed with radial velocity. NGC 6273 has at least two populations separated by $0.2-0.3$ dex in $[\mathrm{Fe} / \mathrm{H}]$. The sodium and aluminum abundances are correlated while the magnesium and aluminum abundances are anti-correlated. The cluster also shows a rise in the abundance of the s-process element lanthanum with $[\mathrm{Fe} / \mathrm{H}]$ similar to other massive clusters. The cluster contains a possible third population depleted in most elements by 0.3 dex.
\end{abstract}

Keywords. globular clusters: individual (NGC 6273), stars: abundances

\section{Introduction}

The Bulge globular cluster NGC 6273 (M19) lies only 1.7 kiloparsecs from the Galactic Center (Harris 1996, 2010 edition), and is among the most massive and luminous globular clusters in the Milky Way. The cluster resembles $\omega$ Centauri in many ways, including its overall metallicity $([\mathrm{Fe} / \mathrm{H}] \approx-1.75)$, its high ellipticity $(\mathrm{e}=0.28)$, and its multi-modal horizontal branch.

As part of a study of clusters in the Galactic Bulge, we obtained high resolution spectra of giant stars in NGC 6273 to examine its composition in more detail and specifically to determine if the cluster's stars exhibit a distribution of metallicities similar to $\omega$ Cen. Spectra were obtained using the Michigan/Magellan Fiber System (M2FS) on the Magellan-Clay 6.5-m telescope at the Las Campanas Observatory.

\section{Results}

Analysis of the spectra reveals a range of $[\mathrm{Fe} / \mathrm{H}]$ abundance among the giants of NGC 6273. The cluster contains an extended metallicity distribution similar to that of $\omega$ Cen, as shown in Figure 1 from Johnson et al. (2015b). We also include metallicity distribution functions for four other clusters with extended metallicity distributions, including $\omega$ Cen (Johnson \& Pilachowski 2010), M22 (Marino et al. 2011), M2 (Yong et al. 2014), and 


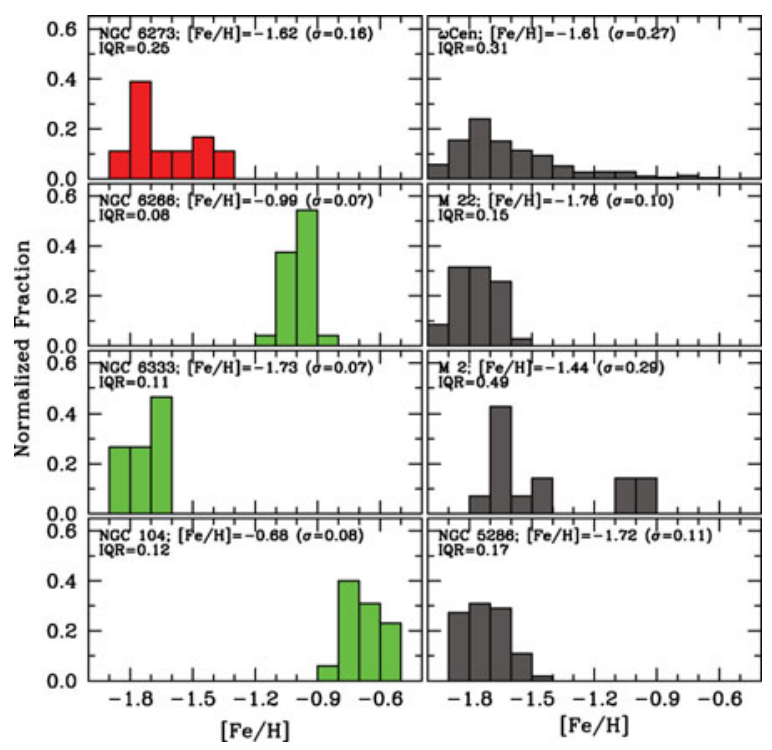

Figure 1. Metallicity distribution function for NGC 6273. Also shown are distribution functions for four globular clusters with a range of observed iron abundances, and for three clusters with no observed spread in $[\mathrm{Fe} / \mathrm{H}]$. See text for references. This figure is reproduced from Johnson et al. (2015b).

NGC 5286 (Marino et al. 2015). We also include three clusters with essentially uniform iron abundances: 47 Tuc (Johnson et al. 2015a), and NGC 6266 and NGC 6333, which were also observed with M2FS.

NGC 6273 shows an extended metallicity distribution function with at least two populations of stars. The width of the metallicity distribution function is highly reminiscent of what is seen in $\omega$ Cen. NGC 6273 appears to contain at least two populations of stars, a metal-poor population centered at $[\mathrm{Fe} / \mathrm{H}]=-1.75$, and a higher metallicity population centered at $[\mathrm{Fe} / \mathrm{H}]=-1.50$. We also found one anomalous star at $[\mathrm{Fe} / \mathrm{H}]=-1.3$ that may indicate the presence of a third population. The anomalous star's composition is also unusual, showing $[\mathrm{m} / \mathrm{Fe}]$ deficiencies of roughly -0.3 dex for most elements.

Element to iron abundance ratios in NGC 6273 also share similarities with $\omega$ Cen, including a pattern of decreasing dispersion in $[\mathrm{Na} / \mathrm{Fe}]$ with increasing metallicity, a bimodal signature in $[\mathrm{Al} / \mathrm{Fe}]$, and an increase in $[\mathrm{La} / \mathrm{Fe}]$ with metallicity. The increase in the $[\mathrm{La} / \mathrm{Eu}]$ ratio suggests the increase in $[\mathrm{La} / \mathrm{Fe}]$ is due to s-process enrichment.

\section{References}

Dotter, A., Chaboyer, B., Jevremović, D. et al. 2008, ApJS, 178, 89

Harris, W. E. 1996 (2010 edition), AJ, 112, 1487

Johnson, C. I. \& Pilachowski, C. A. 2010, ApJ, 722, 1371

Johnson, C. I., McDonald, I., Pilachowski, C. A., et al. 2015a, AJ, 149, 71

Johnson, C. I., Rich, R. M., Pilachowski, C. A., et al. 2015b, AJ, 150:63

Marino, A. F., Sneden, C., Kraft, R. P., et al. 2011, A\&SA, 532, A8

Marino, A. F., Milone, A. P., KaraKas, A. I, et al. 2015, MNRAS, 450, 815

Yong, D., Roderer, I., Grundahl, F. et al. 2014, MNRAS, 441, 3396 